REVIEW.

\title{
A Practical Manual of Diseases of the Skin.*
}

The author states, in his preface, that this work does not profess to be an elaborate treatise on Dermatology, and that it is intended not for the use of the specialist, but for the assistance of the student and general practitioner.

Such being the case, discussions on nomenclature and theoretical speculations on pathology and etiology may be considered to have been advantageously much curtailed. If the nature, course and treatment of a disease be thoroughly known to, and recognized by, the physician, it does not matter very much, either to him or his patient, which of the numerous names applied by the various authorities on the subject he employs to designate the malady; at the same time, such questions as the similarity, or identity, between eczema, seborrhœe and seborrhœea-eczema might have received a passing notice.

The plan adopted, at the commencement, of grouping together the descriptions of the anatomy and physiology, along with the diseases and their treatment, of the various component parts of the integument in a distinct section for each, seems a good one, but it has not been systematically carried out-a particularly striking omission being the absence of a separate chapter on the hair. The histology and physiology of the latter are apparently considered unworthy of notice, as no description of them has been found after a careful perusal of the work. Also, it is difficult to understand why psoriasis, lichen, \&c., should be considered under " inflammations of the skin," while "eczema" receives an entirely separate heading. The only deduction from this is thatthe author does not consider eczema to be an inflammation of the skin; and it is also evident that he does not think it has any

* A Practical Manual of Diseases of the Skin. By George H. Rohé, M.D. \&c., \&c. 
connection with the sebaceous glands, as no mention is made of it in the chapter devoted to the latter.

The book is, avowedly, intended for the use of those who are supposed to know little, if anything, of skin disesses, and these will often have great difficulty in getting a clear and satisfactory comprehension of the subjects considered. To take an example, under "Sycosis" we are, at the commencement of the chapter, informed of the characteristics of non-parasitic sycosis, but we receive no hint as to the nature of parasitic sycosis, or even that such a disease exists, till about three pages farther on, when the following paragraph occurs:- - "From parasitic sycosis the differentiation will be aided by a history of the case. The latter disease usually * commences as a ringworm-tines circinata-and the fungus, which is the cause of the disease, can usually be found, without much difficulty, in the scales, and affected hairs, by the aid of a good microscope," \&c. But we are not told how to find this fungus, nor, having done so, how to recognize it. So far we have not received a word of information regarding the nature and appearances of tinea circinata. Suppose ourselves students, or even young practitioners, anxious for knowledge of this important subject; we eagerly turn up the index for "Parasitic skin diseases," and are there directed to page 199; here we learn that the organisms producing favus, ringworm, and versicolor "belong to the class fungi, and are easily recognizable under a comparatively low power of the microscope (three to five hundred diameters)." What they look like in this situation is left for the imagination or some authority to describe. In another instance it is stated that " another disease which bears great similarity to eczema is the itch;" no definition of the latter, however, is given; and under "scabies" no mention is made that the two terms are synonymous. There is, moreover, no description of the appearance of the sarcoptes.

A fow more details might often have been advantageously given. Under "Urticaria," surely the pigmented form of the disease might have been mentioned, and though the Lichen question is certainly a vexed one at present, a larger space than a page and a half might have been given to the consideration of this important group, of

\footnotetext{
- The italics are our own.
} 
which the author only mentions two varieties, Lichen planus and Lichen ruber acuminatus.

In a work dealing with one branch of medicine only, one expects more details of treatment than are here given; very little is said about baths, and no mention is made of them in the index, which is in other respects incomplete. In cases of prurigo the use of cod-liver oil, either internally or externally, is not even suggested. In the chapter on scabies we are told that, if, after bathing and application of strong sulphur ointment twice daily for three days, the itching returns, we are to repeat the treatment in a few days. The possibility of the sulphur setting up a dermatitis apparently does not present itself to the mind of Dr. Rohé as no word of caution is given on this point.

A statement that it is unnecessary to cut the hair off in cases of acute eczema of the scalp, is followed in the next paragraph by another to the effect that the first indication for the treatment of eczema of the hairy parts of the face, after removal of the scabs, is daily shaving, and no attempt is made to reconcile the apparent incongruity of the two. Glycerine jellies are not suitable in all cases in which their use has been advocated, but it is absurd to ignore them altogether, and the same may be said of Ichthyol and the various plasters which have been so strongly recommended by Unna and his followers.

Lists of formulæ are doubtless useful, but Nos. 24, 30 and 41 all simply contain acetate of potash, fluid extract of taraxacum, and water; rather an embarras de richesse considering how much necessary information on other important points has been withheld. Also many of the numbers on the list do not correspond with those by which they are referred to in the text-a piece of carelessness which a small amount of trouble in supervising the work would have obviated.

On the whole the book cannot be considered as an acquisition to the series of which it forms a number.

A. Home-Douglas. 